\title{
Realization of a Semiconductor-Based Cavity Soliton Laser
}

\author{
Y. Tanguy, T. Ackemann, ${ }^{*}$ and W. J. Firth \\ SUPA, Department of Physics, University of Strathclyde, 107 Rottenrow, Glasgow G4 ONG, Scotland, United Kingdom
}

\section{R. Jäger}

ULM Photonics GmbH, Lise-Meitner-Strasse 13, 89081 Ulm, Germany

(Received 2 July 2007; published 11 January 2008)

\begin{abstract}
The realization of a cavity soliton laser using a vertical-cavity surface-emitting semiconductor gain structure coupled to an external cavity with a frequency-selective element is reported. All-optical control of bistable solitonic emission states representing small microlasers is demonstrated by injection of an external beam. The control scheme is phase insensitive and hence expected to be robust for all-optical processing applications. The mobility of these structures is also demonstrated.
\end{abstract}

Self-localized structures in driven nonequilibrium systems, loosely termed dissipative solitons, have attracted great interest because of their importance in a wide variety of fields (see, e.g., [1] for a recent review). They are particularly interesting in optics - where they are usually referred to as cavity solitons (CSs) - because of potential applications to the all-optical control of light, a major thrust of modern photonics (e.g., [2]). The achievement, control, and understanding of CSs have shown remarkable progress in recent years; see, e.g., [3,4] and Chapters 3-6 of [1]. So far, however, nearly all realized schemes have relied on driving by a broad-area holding beam of high spatial and temporal coherence (e.g., [4-7]). While some laser schemes have been proposed and/or demonstrated to sustain CSs [8-12], no viable cavity soliton laser (CSL) has been developed for a major laser technology. This useful device would convert broad-area excitation into a narrow, coherent, power beam of high quality, or into a controllable number of such beams providing a new approach to microlasers.

Furthermore, like any free-running laser, a lasing CS has the freedom to choose phase, polarization, and frequency, which gives exciting new opportunities for fundamental studies. For example, the relative phase between CSs is expected to affect their dynamics and interaction properties [13], and it will be interesting to compare the effects of phase-sensitive interactions of laser cavity solitons with the wealth of phenomena known for propagating spatial solitons [14]. Similarly, in an isotropic setup, the polarization of the CS becomes a variable to be investigated and maybe utilized. The freedom to chose the operation frequency includes in a long enough laser the potential for multimode oscillation, and perhaps for mode locking. Ultimately, this might lead to spatiotemporal solitons or self-localized optical light bullets $[15,16]$, if the pulse length is shorter than the transit time through the experimental system.

Here we demonstrate optically controlled excitation and erasure of tiny self-localized "lasers" within a semiconductor vertical-cavity surface-emitting laser (VCSEL) structure. Our scheme is easily extensible to all types of VCSELs and is therefore in the mainstream of laser engineering. It could have a substantial impact, both as a fundamentally new type of laser and in the numerous technologies reliant on semiconductor lasers. Semiconductor microcavities are important for photonics applications because of compactness, speed, and ease of integration [4] and CSs have been observed in a variety of configurations, but always involving coherent driving $[4-7,17,18]$. In all these cases, the frequency and phase of the CS is locked to that of the injected field. This enables efficient writing, erasing, and coherent manipulation of CSs [7]. It also requires, however, active phase control within and between devices, and this is a drawback for applications. Hence there are advantages in removing the need for a holding beam and for phase-controlled addressing by moving to a lasing configuration with self-sustained CSs. This also means that the device can draw its energy from an inexpensive, incoherent source (dc current supply in our case). Our realized cavity soliton laser has these important properties.

Cavity soliton laser schemes reported previously used either dye or photorefractive gain media [8-10] and employed intracavity saturable absorbers to favor bistability - a positive indicator for CSs [1,3]- between the lasing and nonlasing states. A semiconductor-based CSL would be much faster, more compact, and more reliable than these systems. A recent theoretical paper considered a semiconductor CSL using a saturable absorber [12]. We adopt a different approach, based on using a VCSEL in conjunction with a frequency-selective external cavity, one attractive feature of it being that it can be implemented with essentially any VCSEL structure using off-the-shelf optical components. Encouragingly, previous experiments have demonstrated bistability in small-area VCSELs in similar feedback configurations [19,20]. Bistability was shown to be due to phase-amplitude coupling [21], by which the different carrier densities of the lasing and nonlasing states imply different refractive indices, and hence 
different cavity resonance frequencies. There can thus be a stable nonlasing state, with the cavity and external (grating-controlled) frequency out of resonance, coexistent with stable in-resonance lasing [19]. Self-localization of the lasing state to form a CS sitting on a nonlasing background requires a nonlinear transverse effect to sustain "gap" states below the band of extended lasing states. Self-focusing in a broad-area VCSEL can supply such an effect. Indeed, CSs have been found in a rather similar model system [22].

The experimental setup is shown in Fig. 1. The VCSEL used is a broad-area bottom-emitting device, emitting at $980 \mathrm{~nm}$ and electrically pumped through a $200 \mu \mathrm{m}$ diameter circular oxide aperture. The epitaxial structure is similar to the one described in $[4,23]$. The self-imaging external cavity includes two lenses and a holographic grating in Littrow configuration. Because of the self-imaging geometry, the high Fresnel number of the solitary VCSEL, and thus the potential for self-localization independent of the boundaries, is preserved. Two beam samplers are also added, one to couple out a fraction of the beam for measurements, and the other to optionally inject a narrow writing beam (WB) from a tunable source.

CSs are self-localized bistable structures in spatiallyextended dissipative systems. This prescription is easy to check in theoretical models, but CS identification in necessarily imperfect experimental schemes is nontrivial. The established procedure (e.g., [4]) is to demonstrate (i) existence at different locations in the transverse plane of localized bistable structures with a well-defined shape and (ii) their mutual independence (e.g., by independent switch-on and switch-off). A further indicator (iii) is then "mobility," i.e., that they can be easily moved around by

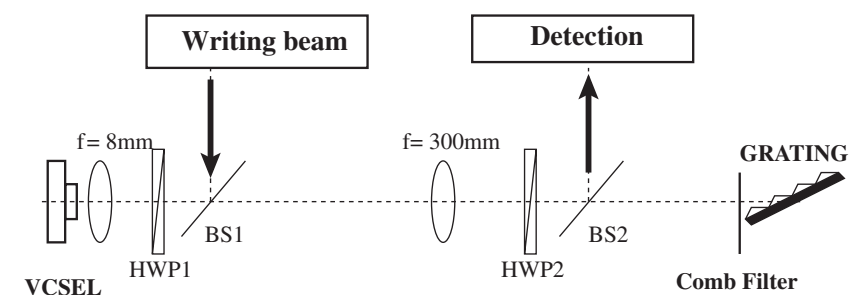

FIG. 1. Experimental apparatus. The external cavity is $616 \mathrm{~mm}$ long, including an $8 \mathrm{~mm}$ aspherical lens used as a collimator and a $300 \mathrm{~mm}$ lens: it is thus self-imaging, projecting a $37.5 \times$ image of the VCSEL onto a Littrow-mounted holographic diffraction grating (1800 lines/mm). Beam sampler BS1 couples a writing beam into the cavity, while BS2 couples out part of the beam for detection. Half-wave plates (HWP) are used to match the principal axes of the VCSEL, the intracavity beam splitters, and the grating. The strong polarization dependence of the grating's diffraction efficiency ensures that the intracavity field has a well-defined linear polarization. Hence the HWPs can also be used to control the coupling efficiency of the writing beam and of the detection arm to the external cavity. For some measurements a comb filter is added in the reimaged near field close to the grating. Its slits are orthogonal to the grating lines. perturbations and hence are independent of boundary conditions and self-localized. In order to claim a CSL, the transition (iv) to narrow-band coherent emission obviously needs to be shown also.

We demonstrate fulfilment of criteria (i) and (iv) by studying the behavior of the near field under variation of the injected current. The VCSEL is biased below the threshold of the solitary laser, and the grating is aligned so that its frequency of maximal feedback is red-detuned with respect to the longitudinal resonance frequency of the VCSEL (cf. also Ref. [19]). Increasing the injection current, we observe spontaneous formation of several localized spots, all of similar size and brightness, with a diameter of about $10 \mu \mathrm{m}$ (FWHM; see the upper right panels in Fig. 2). These spots display bistability in dependence on current, with abrupt switch on and off (see Fig. 2, left panel). Measurements with a scanning Fabry-Perot interferometer indicate that a spot can, depending on parameters such as current and temperature, operate on one or more longitudinal modes of the external cavity (which are separated by about $240 \mathrm{MHz}$ ). The single-mode emission linewidth is $10 \mathrm{MHz}$; i.e., each of these spots is a tiny laser emitting coherent light.

Regarding (ii), independence and simultaneous bistability of two of these spot lasers is demonstrated by independent switch on and switch off by an injected field. The sequence is shown in Fig. 3. The WB is incoherent with the spots, so that the switching is phase insensitive. The mechanism is discussed below. We demonstrate (iii), mobility of these microlasers, by perturbing them with the WB injected at some distance from the spot. They move toward it and can be dragged around with it for significant distances (several beam diameters).

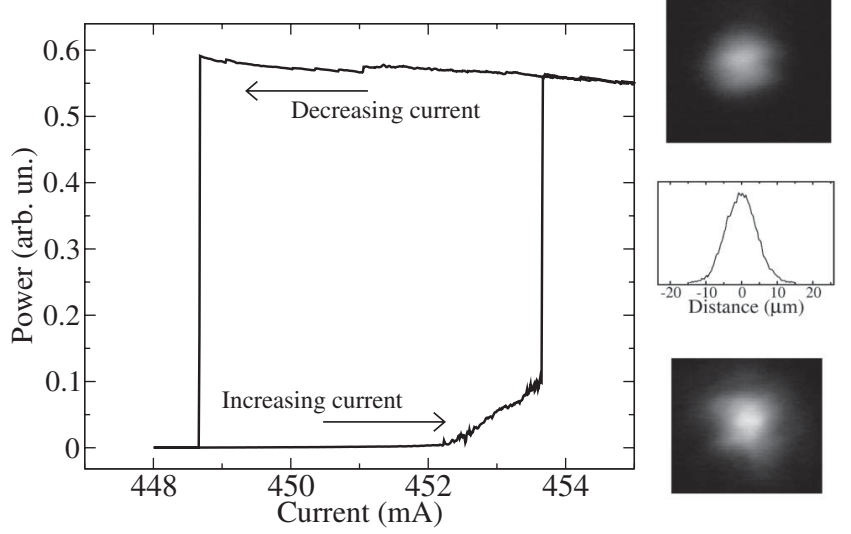

FIG. 2. Power versus current for a single spot. In this case the comb filter (explained below) was inserted in order to reduce the optical background of the spot and hence obtain a cleaner hysteresis. The panels on the right show, from top to bottom, the near-field intensity distribution around the spot, a transverse intensity profile through the center of the near-field distribution, and the far-field intensity distribution. 


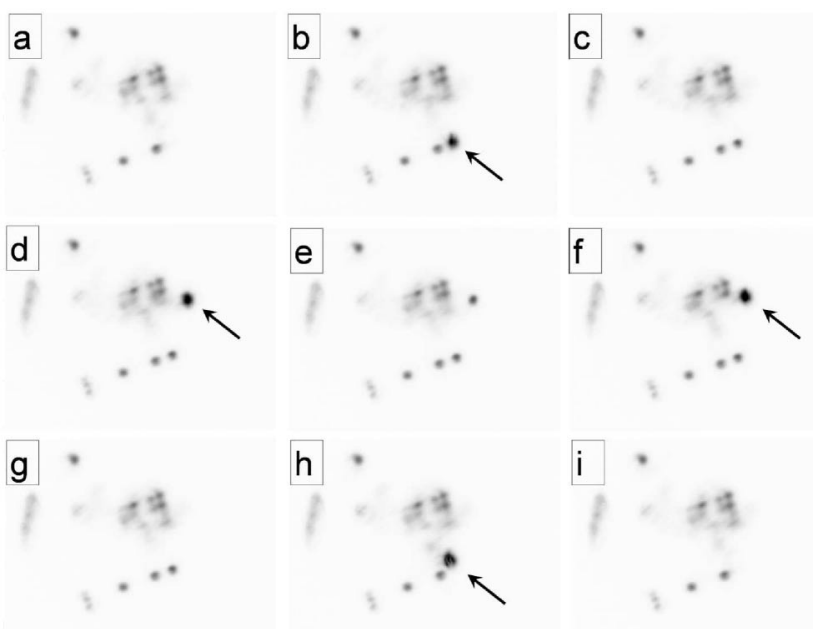

FIG. 3. Near field intensity distributions showing the successive switching on and off of two spots with an injected incoherent field (brightest spots, indicated by arrows). Dark areas correspond to high intensities. The WB is derived from a tunable laser source, with wavelength tuned in the vicinity of the VCSEL cavity resonance. It is focused onto the VCSEL with a $12 \mu \mathrm{m}$ spot diameter (FWHM) and a power in the $\mathrm{mW}$ range. Two sites where spontaneous spots could be observed were selected for WB injection, and the VCSEL was biased within their bistability range. (a) Both spots are off, (b) injection of WB, (c) one spot is switched on and remains after the WB is blocked, (d) injection of WB at a second location, (e) second spot remains on, (f) WB injected beside second spot, (g) second spot switched off and does not reappear (first spot unaffected), (h) injection of writing beam to switch off first spot, and (i) both spots remain off.

This set of observations establishes that our device is indeed a cavity soliton laser and opens up the all-optical control of microlasers on demand.

Recorded spectra showed that, for fixed current and temperature, the single-mode frequency of a specific CS fluctuated over a range of about $2 \mathrm{GHz}$. This can be interpreted on the basis that there is a family of CS solutions, each associated with different longitudinal modes of the external cavity. The finite frequency selectivity of the feedback enables several different family members to be excited by the WB and permits noise-driven jumps between them. Thus there is no "memory" of the WB frequency, which is therefore only activating CS lasing, not determining the CS properties.

Figure 2 shows that some emission is already present (above $452 \mathrm{~mA}$ ) before the CS actually switches on. This emission comes from low amplitude background states or patterns, visible also in Fig. 3. These are blue-detuned by some tens of GHz to the CS and their linewidth is considerably larger. Their excitation is attributed to the finite frequency selectivity of the system. This optical background seems to be detrimental to the existence of the CS. In order to reduce it, a comb filter was inserted in the external Littrow cavity, positioned as close as possible to the reimaged near field at the grating (Fig. 1). This filter allows feedback only on several horizontal stripes and thus creates a near one-dimensional CS confinement. Figure 4 shows the resulting near field with this filter, where the optical background is clearly weakened and more CSs have emerged. The characteristic in Fig. 2, taken with a comb filter, shows an extended region at lower current where there is coexistence of the CSs and a background-free state (the off state of the VCSEL).

Moving the comb filter vertically gives further evidence for CS mobility (see the other panels in Fig. 4, between which several CSs clearly change position). Within some areas of the sample, their shift is quasicontinuous; in others they seem to "jump" from one preferred location to the other. Their mobility distinguishes CSs from fundamental modes of bistable small-area laser defined, e.g., by micromachining. Its utilization enables new features as alloptical delay lines, optically controlled beam steering, and self-alignment to different kinds of external inputs.

The experiments shown in Fig. 4 reveal also (as do the images in Fig. 3) that the CSs do not have complete freedom of location in the present device, but tend to be attracted to, and trapped by, certain "defects." Trapping effects were also found in other CS systems based on similar VCSELs $[4,18]$. These defects are no doubt due to local inhomogeneities in the active layer (temperature or carrier density inhomogeneities) and/or the mirror layers (index inhomogeneities). The sensitivity of the CS location to such imperfections suggests a diagnostic application.

There is a novel and interesting self-induced force acting on the CS in our system. The GHz spread of CS frequencies means the emission frequency cannot be locked to the grating's peak feedback frequency (see also [19]). Any detuned beam is fed back at a slightly different angle from its emission, which induces a phase gradient (of $2.9 \mathrm{mrad} / \mu \mathrm{m} / \mathrm{GHz}$ ) across the CSs. Phase gradients are well known from other CS systems to exert forces, leading to CS drift in an otherwise homogeneous system [24]. This means that the preferred CS locations in our system are actually equilibria between defect and grating-induced

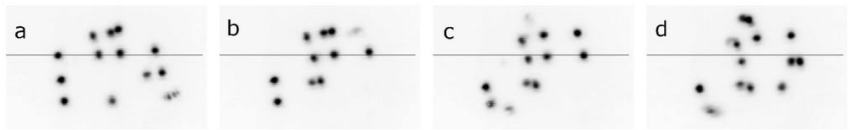

FIG. 4. Near field intensity distributions displaying the effect of a comb filter placed in the external cavity at the reimaged near-field position. Dark areas correspond to high intensities. This filter allows feedback only on several horizontal stripes with width $16 \mu \mathrm{m}$ at the VCSEL, spaced by the same distance. From (a) to (d) the comb filter is moved downward. A line is added for reference, clearly showing that CS positions are shifted between panels. For example, the rightmost CS close to the line in (a) shifts quasicontinuously by about $18 \mu \mathrm{m}$ between (a) and (d). Some CSs appear and disappear spontaneously, as a result of local inhomogeneities. 
forces. We confirmed the presence of a significant tilt force by reversing the orientation of the grating, which led to small shifts in all the preferred CS locations. The directions of these shifts are consistent with the tilt-force model, and their signs indicate that all the CSs are blue-detuned from the grating peak; i.e., the CS frequencies lie between the grating and VCSEL frequencies. Direct experimental measurement of the tilt is quite difficult, however, because it seems to be much smaller than the angular far-field width of a CS, typically 44 mrad.

The switching behavior shown in Fig. 3 is also consistent with the defect model including the grating-induced force. Switch on works well with the WB directly on the target location. For switch off the WB is positioned to the side, so as to perturb the trap and initiate erasure, probably via carrier effects. However, switch off is not possible for every orientation of the WB with respect to the CS. When the phase gradient is oriented so as to direct the CS toward the trap, the CS reappears after the WB is removed. At these orientations switch on is possible, however. Reversing the grating, and thus reversing the direction of the phase gradient, swaps these locations to the other side of the CS.

The switching sequence shown in Fig. 3 was performed with quasi-cw beams (i.e., by mechanically opening and closing the beam path of the WB for some seconds) because in that case the injection is easily visualized. However, switch on and switch off are also possible with a pulsed WB. The minimum pulse length investigated is $25 \mathrm{~ns}$, with switch delays in the $10 \mathrm{~ns}$ range, and is limited by the available acousto-optic modulator [25]. Polarization and frequency detuning between CS and WB are not critical but will influence the necessary switching energy and the resulting time delay.

In conclusion, we have demonstrated cavity soliton microlasers in a semiconductor-based system and their all-optical control. The control is incoherent; i.e., there are no stringent phase or frequency requirements on the external beams used for writing, erasing, or manipulation of the CSs. This makes the scheme robust for applications. We expect that switching times are currently limited by both the external cavity round-trip time and carrier relaxation. The latter could be improved by engineering of the carrier lifetime $[26,27]$. With regard to round-trip time, we have preliminary evidence of CS in a short cavity closed by a volume Bragg grating [28]. Thus there is every prospect that use of micro-optics (potentially monolithic integration) will allow significant improvements in both speed and compactness of these devices.

This work was supported by the EU STREP FunFACS (www.funfacs.org) and by the Faculty of Science of the
University of Strathclyde. Discussions with J. R. Tredicce stimulated this work, while M. Sondermann and F. Marino contributed to preliminary experiments. We are grateful to FunFACS partners, and to P. Paulau, N. A. Loiko, and A. V. Naumenko, for many useful discussions and suggestions.

*thorsten.ackemann@strath.ac.uk

[1] Dissipative Solitons, Lecture Notes in Physics Vol. 661, edited by N. Akhmediev and A. Ankiewicz (Springer, New York, 2005).

[2] D. Cotter et al., Science 286, 1523 (1999).

[3] L. A. Lugiato, IEEE J. Quantum Electron. 39, 193 (2003).

[4] S. Barland et al., Nature (London) 419, 699 (2002).

[5] V. B. Taranenko and C. O. Weiss, Appl. Phys. B 72, 893 (2001).

[6] S. Barbay et al., Opt. Lett. 31, 1504 (2006).

[7] X. Hachair et al., Phys. Rev. A 72, 013815 (2005).

[8] V. Y. Bazhenov, V. B. Taranenko, and M. V. Vasnetsov, Proc. SPIE-Int. Soc. Opt. Eng. 1840, 183 (1992).

[9] M. Saffman, D. Montgomery, and D.Z. Anderson, Opt. Lett. 19, 518 (1994).

[10] V.B. Taranenko, K. Staliunas, and C. O. Weiss, Phys. Rev. A 56, 1582 (1997).

[11] N. N. Rosanov, Spatial Hysteresis and Optical Patterns, Springer Series in Synergetics (Springer, Berlin, 2002).

[12] M. Bache et al., Appl. Phys. B 81, 913 (2005).

[13] N. N. Rosanov, S. V. Fedorov, and A. N. Shatsev, Phys. Rev. Lett. 95, 053903 (2005).

[14] G. I. Stegeman and M. Segev, Science 286, 1518 (1999).

[15] F. W. Wise and P. Di Trapani, Opt. Photonics News 13, No. 2, 28 (2002).

[16] M. Brambilla, T. Maggipinto, G. Patera, and L. Columbo, Phys. Rev. Lett. 93, 203901 (2004).

[17] Y. Larionova and C. Weiss, Opt. Express 13, 10711 (2005).

[18] X. Hachair et al., IEEE J. Sel. Top. Quantum Electron. 12, 339 (2006).

[19] A. Naumenko et al., Opt. Commun. 259, 823 (2006).

[20] Y. Tanguy, T. Ackemann, and R. Jäger, Phys. Rev. A 74, 053824 (2006).

[21] C. H. Henry, IEEE J. Quantum Electron. 18, 259 (1982).

[22] P. Paulau et al., Phys. Rev. E 75, 056208 (2007).

[23] M. Grabherr et al., IEEE J. Sel. Top. Quantum Electron. 5, 495 (1999).

[24] W. J. Firth and A. J. Scroggie, Phys. Rev. Lett. 76, 1623 (1996).

[25] Y. Tanguy, T. Ackemann, and R. Jäger, Opt. Express 15, 16773 (2007).

[26] A. Garnache et al., Appl. Phys. Lett. 80, 3892 (2002).

[27] E. A. Avrutin, J. H. Marsh, and E. L. Portnoi, IEE Proc.Optoelectronics 147, 251 (2000).

[28] N. Radwell, Y. Tanguy, and T. Ackemann (unpublished). 